\title{
RR Lyrae Variables in the Second-Parameter Globular Cluster NGC 7006
}

\author{
Amelia Wehlau ${ }^{1} \&$ James M. Nemec ${ }^{2}$ \\ ${ }^{1}$ Dept. of Astronomy, University of Western ONtario, London ON N6A3K7 Canada \\ ${ }^{2}$ Program in Astronomy, Washington State University, Pullman WA 99164 USA
}

\begin{abstract}
The distant globular cluster NGC7006 was one of the first clusters studied for which the distribution of stars along the horizontal branch of its C-M diagram showed evidence for a "second parameter" in addition to metallicity. Studies of the more than 60 known RR Lyrae stars in this cluster should yield some statistically significant trends or correlations which might help to identify the second parameter. In the first stage of this study (Wehlau, Nemec, Hanlan \$ Rich 1992, AJ, 103, 1583) photographic data from 1984 were combined with previously published data from the 1930's and 1950's and used to obtain period change rates for 46 variables. The median rate was found to fall one standard error below that predicted by the Yale evolutionary HB models. In addition, statistically significant evidence was found for a radial gradient in period change rates in the sense that rates for variables in the outer region of the cluster were more negative.

In the second stage of the investigation $B$ and $V$ magnitudes derived from CCD frames of the cluster are being used to obtain colors and to increase the number of variables for which good periods are known, in particular variables in the inner region of this centrally concentrated cluster.
\end{abstract}

